Article

\title{
The Positive Effects of Grifola frondosa Heteropolysaccharide on NAFLD and Regulation of the Gut Microbiota
}

\author{
Xin Li ${ }^{1,2}$, Feng Zeng ${ }^{2}$, Yifan Huang ${ }^{1,2,3, *}$ and Bin Liu 1,2,* \\ 1 National Engineering Research Center of JUNCAO Technology, Fujian Agriculture and Forestry University, \\ Fuzhou 350002, China; lixin1229@hotmail.com \\ 2 College of Food Science, Fujian Agriculture and Forestry University, Fuzhou 350002, China; \\ fengzengfz@163.com \\ 3 College of Animal Science, Fujian Agriculture and Forestry University, Fuzhou 350002, China \\ * Correspondence: zjhyfang@163.com (Y.H.); liubin618@hotmail.com (B.L.)
}

Received: 7 September 2019; Accepted: 21 October 2019; Published: 24 October 2019

\begin{abstract}
Non-alcoholic fatty liver disease (NAFLD) is a major public health problem in many countries. In this study, the ability of Grifola frondosa heteropolysaccharide (GFP) to ameliorate NAFLD was investigated in rats fed a high-fat diet (HFD). The molecular mechanisms modulating the expression of specific gene members related to lipid synthesis and conversion, cholesterol metabolism, and inflammation pathways were determined. The components of the intestinal microflora in rats were analyzed by high-throughput next-generation $16 \mathrm{~S}$ rRNA gene sequencing. Supplementation with GFP significantly increased the proportions of Allobaculum, Bacteroides, and Bifidobacterium and decreased the proportions of Acetatifactor, Alistipes, Flavonifractor, Paraprevotella, and Oscillibacter. In addition, Alistipes, Flavonifractor, and Oscillibacter were shown to be significant cecal microbiota according to the Spearman's correlation test between the gut microbiota and biomedical assays $(|r|>0.7)$. Histological analysis and biomedical assays showed that GFP treatments could significantly protect against NAFLD. In addition, Alistipes, Flavonifractor, and Oscillibacter may play vital roles in the prevention of NAFLD. These results suggest that GFP could be used as a functional material to regulate the gut microbiota of NAFLD individuals.
\end{abstract}

Keywords: Grifola frondosa; NAFLD; gut microbiota

\section{Introduction}

A high-fat diet (HFD) is a risk factor for a range of diseases, including lipid metabolism disorders (LMDs), non-alcoholic fatty liver disease (NAFLD), and type-2 diabetes (T2D) [1,2]. NAFLD is currently the most prevalent chronic liver disease worldwide and has become a major public health problem in many countries [2,3]. The majority of NAFLD patients do not recover from this condition; rather, the disease progresses to non-alcoholic steatohepatitis (NASH), hepatocyte fibrosis, cirrhosis, or even hepatocellular carcinoma which are responsible for most deaths worldwide [4]. However, the factors involved in the induction and progression of these disorders are complex and not fully understood [5].

NAFLD is usually considered to be the hepatic manifestation of metabolic syndrome [6]. However, some clinical data have shown that the composition and characteristics of the intestinal flora in patients with metabolic disorders differ from those in normal populations [7,8]. For example, a significant correlation has been shown between the relative abundance of intestinal flora and hepatic dyslipidemia and inflammation $[9,10]$. Currently, a lot of drugs which have been tested in clinical trials could not provide satisfactory results in terms of therapeutic efficacy and side effect. In addition, only a few of these drugs target the intestinal flora in NAFLD. Therefore, it is necessary to search for a novel drug or 
natural compound without any side effects and find out how the compound relates to the intestinal flora in the treatment of NAFLD. In recent years, many polysaccharides with antihyperlipidemic, hepatoprotective, and antioxidant activities have been studied as dietary supplements or functional foods in the treatment of NAFLD [11-14]. However, it is generally known that polysaccharides are biological macromolecules and cannot be directly absorbed and utilized [15]. Fortunately, a previous study has reported that many polysaccharides could be utilized by the intestinal flora [16]. Therefore, the intestinal flora may be a major factor in the mechanisms of the polysaccharides and the relationship between the effect of polysaccharides and intestinal flora in the treatment of NAFLD still remains to be further studied.

Polysaccharides come from a wide range of sources, including animals, plants, fungus, and others [17]. Grifola frondosa is an important medicinal fungus in China and Japan, with diverse bioactive metabolites [18]. The polysaccharide extracted from Grifola frondosa has various biological and pharmacological activities, such as antioxidant, anti-inflammatory, immune-regulating, and antitumor activities $[19,20]$. Although its consumption is generally accepted to be beneficial for health, its mechanisms and relationships with NAFLD and the gut microbiota have not been completely investigated. Therefore, this research sought to assess the potential of Grifola frondosa heteropolysaccharide (GFP) supplementation in ameliorating NAFLD in Wistar rats fed HFD and to investigate the relationship of this mushroom with NAFLD-associated parameters and the gut microbiota.

\section{Results}

\subsection{Effects of GFP on Body Weight and Biochemical Values of NAFLD Rats}

A steep increase was observed in the bodyweights of all rats (Table 1), with the weights of HFD rats increasing most significantly after four $(p<0.05)$ and eight weeks $(p<0.01)$. Animals in the GFP group showed obviously lower body weights than those in the HFD group after four and eight weeks $(p<0.01)$. The bodyweight gain of animals in the GFP group $(154.33 \pm 32.75 \mathrm{~g})$ was significantly lower than that in the HFD group after eight weeks $(p<0.01)$, while there was no significant difference between the GFP and control diet (NFD) group.Increased alanine transaminase (ALT), serum aspartate transaminase (AST) lipid triglycerides (TG), and total cholesterol (TC), and low-density lipoprotein cholesterol (LDL-C) concentrations and decreased liver glutathione peroxidase (GSH-Px), and liver superoxide dismutase (SOD) and the levels of high-density lipoprotein cholesterol (HDL-C) concentrations were the primary features of NAFLD status of rats in the HFD group [21]. However, GFP was shown to significantly attenuate this process in all respects, except for ALT levels (Figure 1). These results indicate the ability of GFP to improve the obesity, liver function, and lipid levels of NAFLD rats.

Table 1. Effects of GFP on body weight and body weight gain as compared withNFD and HFD.

\begin{tabular}{cccc}
\hline \multirow{2}{*}{ Time } & \multicolumn{3}{c}{ Weight $(\mathrm{g})$} \\
\cline { 2 - 4 } & NFD & HFD & GFP \\
\hline 0 weeks & $222.86 \pm 11.1$ & $230.79 \pm 5.93$ & $229.98 \pm 8.03$ \\
4 weeks & $376.75 \pm 31.65$ & $409.78 \pm 27.53^{*}$ & $362.1 \pm 27.67^{\text {\#\# }}$ \\
8 weeks & $388.74 \pm 30$ & $442.73 \pm 33.22^{* *}$ & $384.31 \pm 30.97^{\# \#}$ \\
weight gain & $165.88 \pm 22.05$ & $211.95 \pm 32.88^{* *}$ & $154.33 \pm 32.75^{\# \#}$ \\
\hline
\end{tabular}

Values are presented as the mean $\pm \mathrm{SD}(n=8)$. Differences were assessed by ANOVA and are denoted as follows: ${ }^{*} p<0.05$ versus the NFD group; ${ }^{* *} p<0.01$ versus the NFD group; and \#\# $p<0.01$ versus the HFD group. 
A

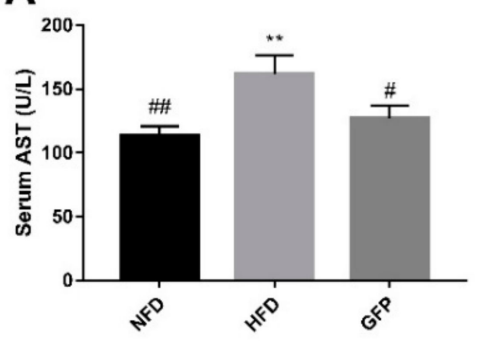

C

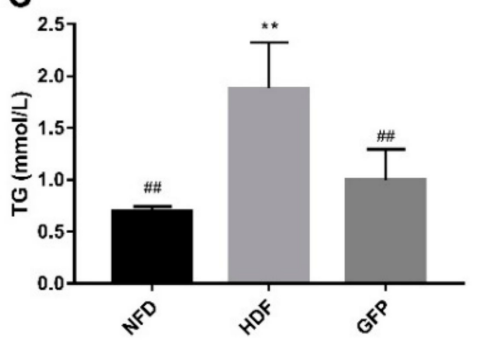

$E$

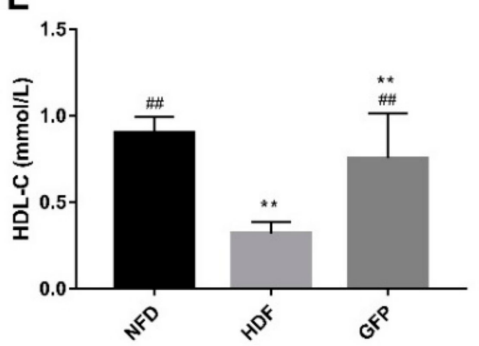

G

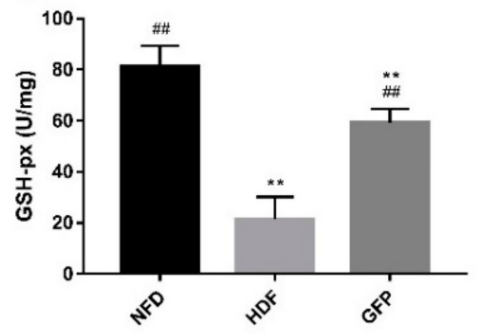

B

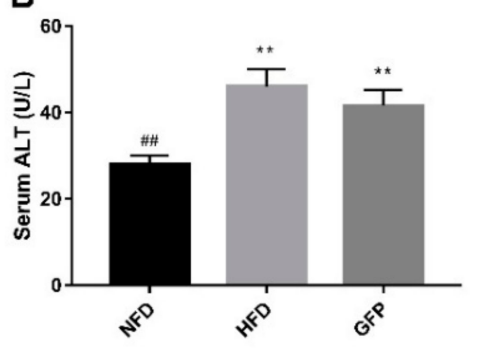

D

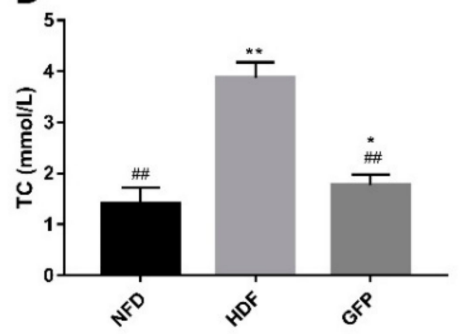

$\mathbf{F}$

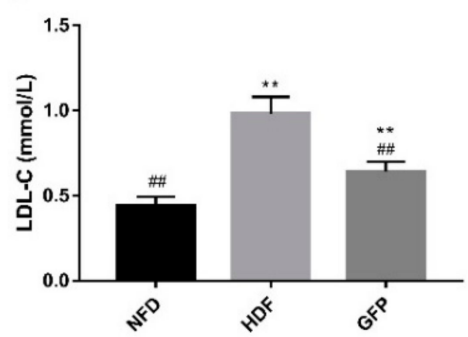

H

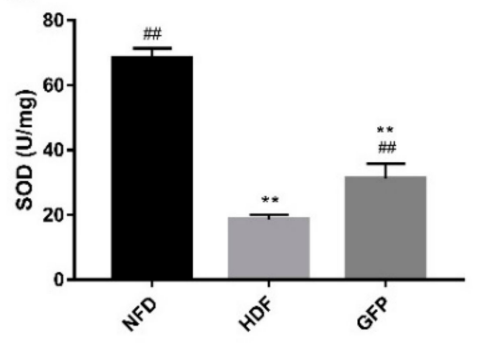

Figure 1. Effects of GFP on liver protection and lipid levels of non-alcoholic fatty liver disease (NAFLD) rats: (A) serum aspartate transaminase (AST); (B) serum alanine transaminase (ALT); (C) lipid triglycerides (TG); (D) total cholesterol (TC); (E) high-density lipoprotein cholesterol (HDL-C); (F) low-density lipoprotein cholesterol (LDL-C); (G) liver glutathione peroxidase (GSH-px); and (H) liver superoxide dismutase (SOD). Values are presented as the mean $\pm \mathrm{SD}(n=5)$. Differences were assessed by ANOVA and are denoted as follows: ${ }^{*} p<0.05$ versus the NFD group; $\# p<0.05$ versus the HFD group; ${ }^{* *} p<0.01$ versus the NFD group; and \# $p<0.01$ versus the HFD group. 


\subsection{Effect of GFP on the Histopathology of NAFLD Rats}

The histopathological changes in hepatic tissue were examined by light microscopy. As shown in Figure 2, liver sections from normal rats showed distinct hepatic cells with abundant cytoplasm, prominent nuclei, and distinct nucleoli. Neither macrovesicular steatosis nor hepatocellular injury was observed. The hepatic cords radiated in regular rays from the central vein in all directions. In contrast, liver tissues from rats in the HFD group exhibited massive fat droplets in the cytoplasm of hepatocytes, and severe steatosis ( $>60 \%$ of hepatocytes involved) was found in the HFD group, with a high macrovesicular component. However, in the GFP group, steatosis reversal was detected with less than 5\% macrovesicular steatosis retained [21,22]. The histological scores of liver sections are presented in Table 2 [23]. The GFP group had significantly reduced hepatocyte steatosis and liver cell injury compared with the HFD group $(p<0.01$ and $p<0.05)$. However, there was no significant difference in lobular inflammation in the GFP group compared with the HFD group.
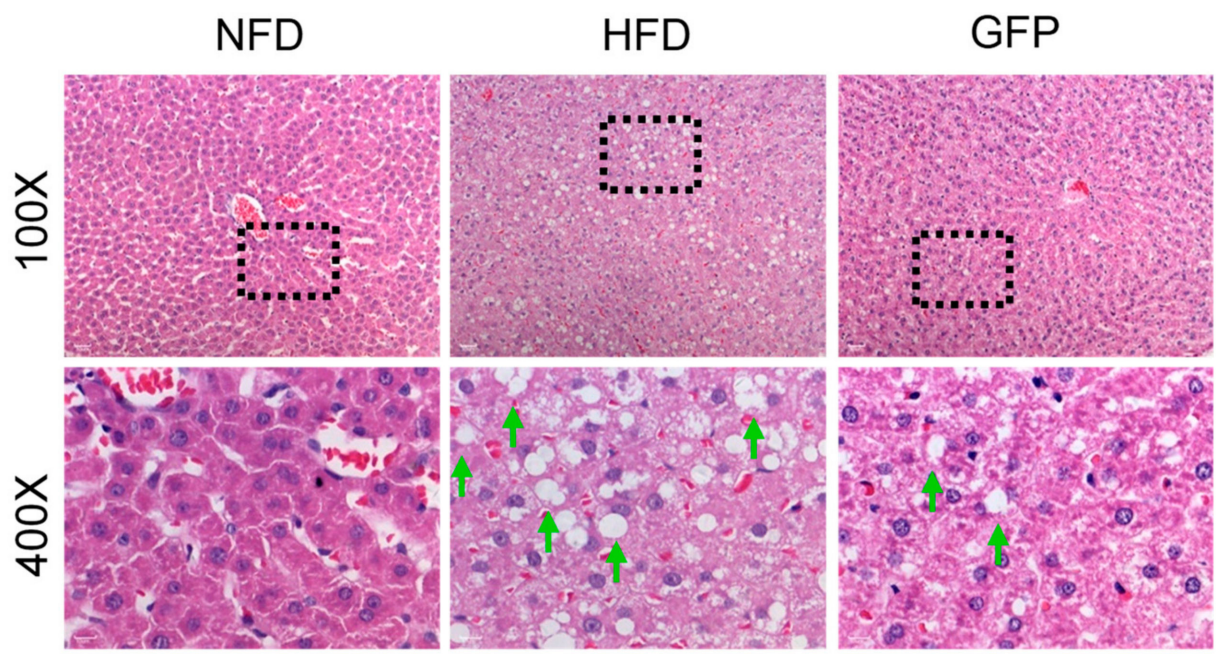

Figure 2. Photomicrographs of rat liver tissues (hematoxylin-eosin staining, 100× and 400×) and histological scores of liver sections. Representative pictures of different groups and higher magnifications of the black dotted boxes in the lower row (green arrows: macrovesicular steatosis).

Table 2. The histological scores of liver sections of GFP group as compared with NFD and HFD groups.

\begin{tabular}{cccc}
\hline Histological Scores & Steatosis Grade & Lobular Inflammation & Liver Cell Injury Ballooning \\
\hline NFD & $0 \pm 0$ & $0 \pm 0$ & $0.4 \pm 0.55$ \\
HFD & $2.4 \pm 0.55^{* *}$ & $0.4 \pm 0.55$ & $2 \pm 0 *$ \\
GFP & $0.8 \pm 0.45^{\# \#}$ & $0.2 \pm 0.45$ & $1.6 \pm 0.55^{*}$ \\
\hline
\end{tabular}

Values are presented as the mean $\pm \mathrm{SD}(n=5)$. Differences were assessed by ANOVA and are denoted as follows: ${ }^{*} p<0.05$ versus the NFD group; ${ }^{* *} p<0.01$ versus the NFD group; and \#\# $p<0.01$ versus the HFD group.

\subsection{Effect of GFP on the mRNA Expression of Genes Involved in NAFLD}

To elucidate the molecular mechanism by which GFP ameliorates HFD-induced NAFLD, the hepatic mRNA expression levels of genes involved in lipid metabolism, oxidative stress, and inflammatory cascades were quantified by RT-qPCR. The administration of HFD resulted in elevations in the mRNA levels of CYP8B1, SOCS2, CYP4A1, CYP4A2, CYP4A3, TNF- $\alpha$, and ACC and a reduction in $C Y P 7 A 1$ gene expression, as shown in Figure $3(p<0.05)$. However, GFP treatment significantly reduced the expression of CYP4A1, ACC, TNF- $\alpha$, and SOCS2 genes $(p<0.05$ or $p<0.01)$, and upregulated the expression of CYP7A1 compared with the HFD group $(p<0.01)$ (Figure 3). Taken together, the above results demonstrate that GFP ameliorates NAFLD by regulating CYP4A1, ACC, 
TNF- $\alpha$, SOCS2, and CYP7A1. These genes play roles in fatty acid oxidation, lipid synthesis, bile acid anabolism, and inflammatory cytokine activity.

CYP7A1

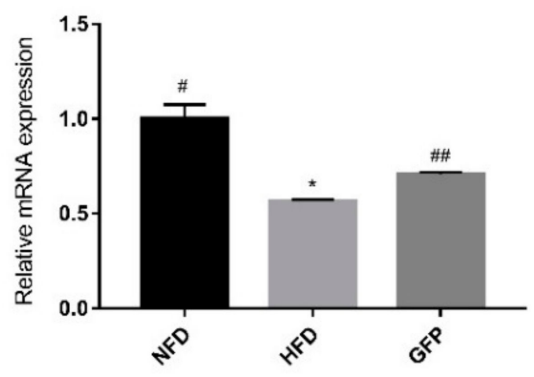

CYP4A1

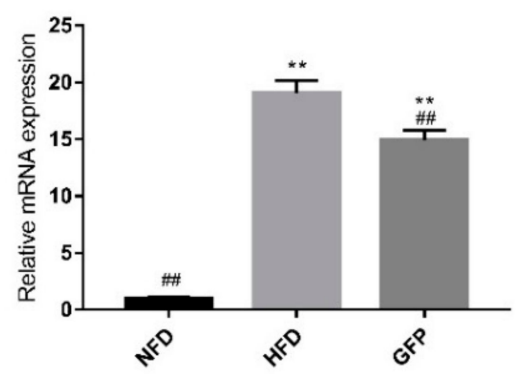

CYP4A3

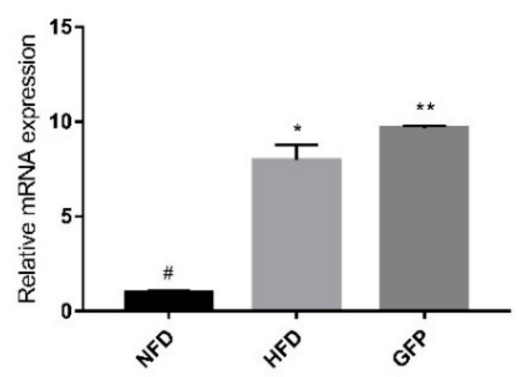

TNFa

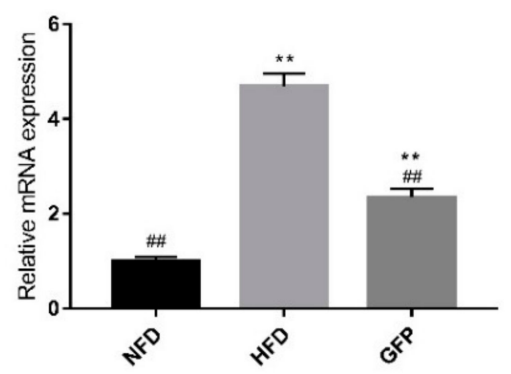

CYP8B1

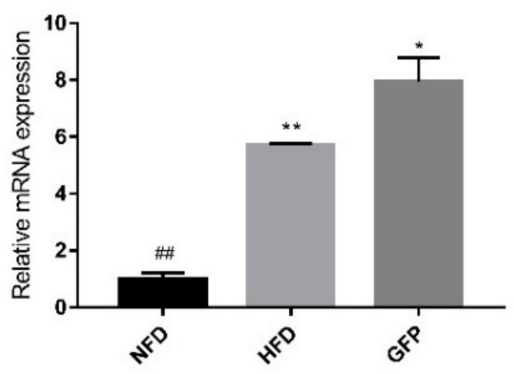

CYP4A2

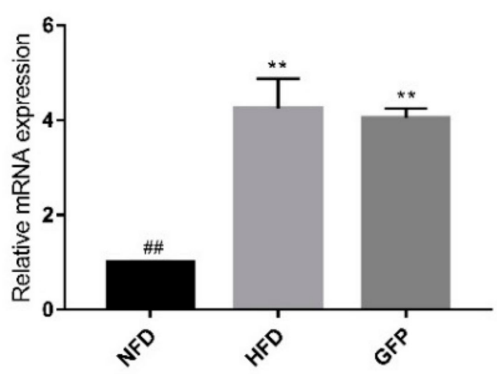

socs2

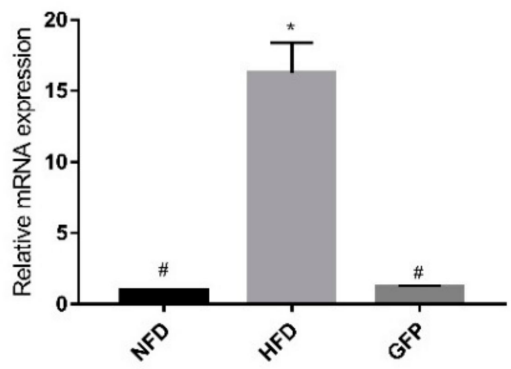

ACC

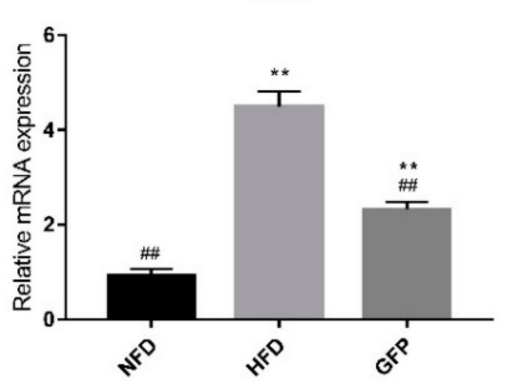

Figure 3. Effect of GFP on the mRNA expression of genes involved in NAFLD as determined by real-time PCR. $\beta$-actin was used as a reference gene. Data are presented as the mean $\pm \mathrm{SD}, n=3$. Differences are denoted on the data labels as follows: ${ }^{*} p<0.05$ and ${ }^{* *} p<0.01$ compared with the NFD group; $\# p<0.05$ and \#\# $p<0.01$ compared with the HFD group. 


\subsection{Effects of GFP on the Community Structure of the Gut Microbiota in NAFLD Rats}

To investigate the effects of the treatments on the community structure of the gut microbiota, the bacterial communities were analyzed at the phylum level (Figure 4). The abundance of Firmicutes was increased, while Bacteroidetes were significantly decreased in the HFD group compared with the NFD group. Compared with the HFD group, the GFP groups exhibited a significant increase in Bacteroidetes and a significant decrease in Firmicutes.

A

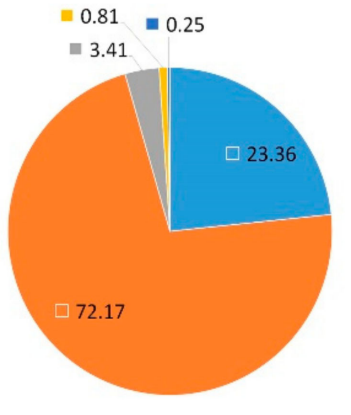

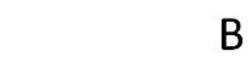

- Bacteroidetes

m Firmicutes

- Proteobacteria

Actinobacteria

- Others

B

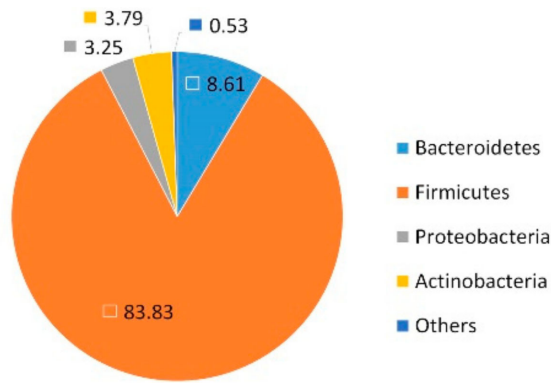

C

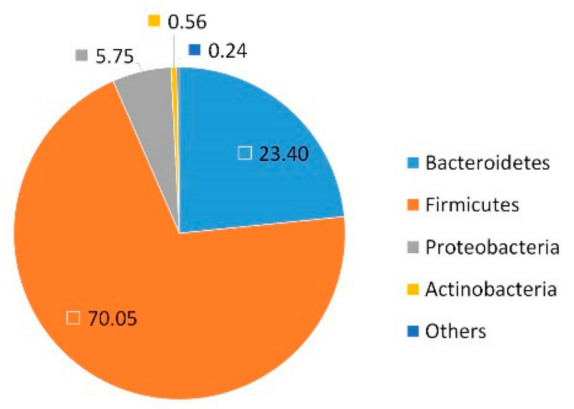

Figure 4. Fecal bacteria at the phylum level based on relative abundance. The color and number correspond to the normalized relative abundance (percent) of the taxa at the phylum level: (A) the NFD group; (B) the HFD group; and (C) the GFP group.

The gut microbiota analysis at the genus level revealed HFD-induced changes in the composition of the gut microbiota. Our results show that the relative abundances of Allobaculum, Bacteroides, Bifidobacterium, Blautia, Coprococcus, Phascolarctobacterium, Prevotella, and Roseburia decreased in the HFD group compared with the NFD group. Conversely, the relative abundances of Acetatifactor, Alistipes, Flavonifractor, Paraprevotella, and Oscillibacter significantly increased in the HFD group compared with the NFD group. The HFD-induced changes in the cecal microbiota structure were partly reversed by GFP (Figure 5).

To assess the potential relationship between GFP-induced changes in gut microbiota composition and biochemical indicator levels, Spearman's correlation analysis was carried out (Figure 6A). The results of Spearman's correlation analysis showed that the abundance of bacteria belonging to Acetatifactor, Alistipes, Flavonifractor, Paraprevotella, and Oscillibacter, which were decreased in the GFP compared with the HFD group, were positively correlated with AST, ALT, TC, TG, and LDL-C concentrations, but negatively correlated with GSH-Px, SOD, and HDL-C levels. A visualization of the correlation network based on the significant correlations between the cecal microbiota and the biochemical parameters was constructed (Figure 6B). According to Spearman's correlation test $(|r|>0.7)$, significant cecal microbiota, such as Flavonifractor, Oscillibacter, and Alistipes, are included in the network. 


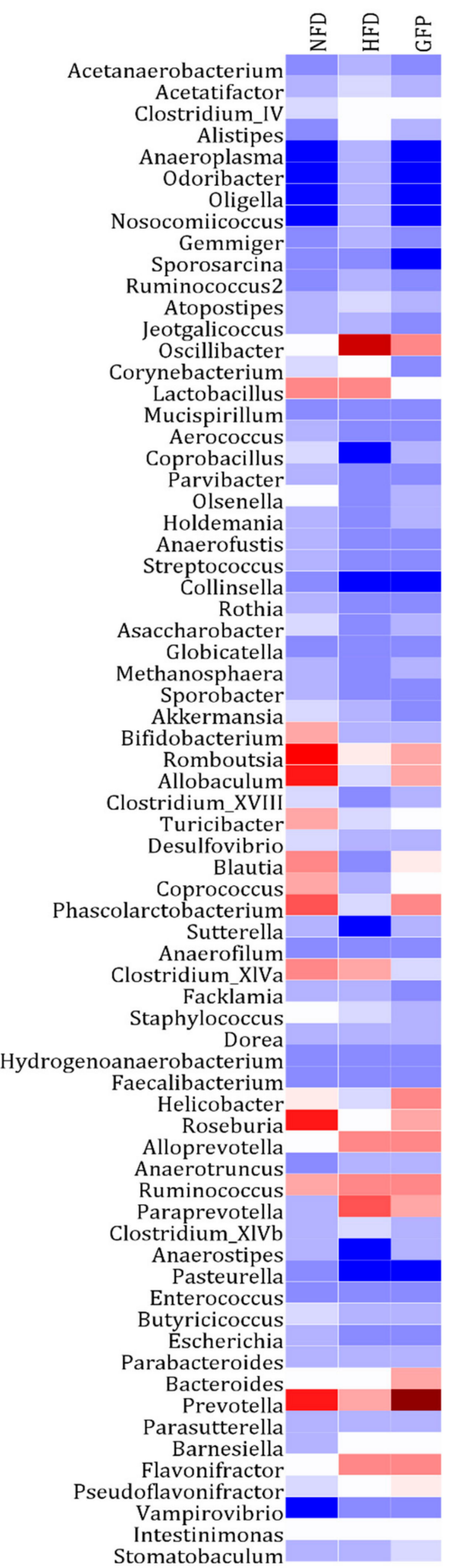

Figure 5. Heatmap of fecal bacterial taxa at the genus level based on relative abundance. The color of each spot corresponds to the normalized relative abundance (percent) of the taxa at the genus level. The genus names of the taxa are shown on the left. The fecal samples from the NFD, HFD, and GFP groups are shown on the top. 
A

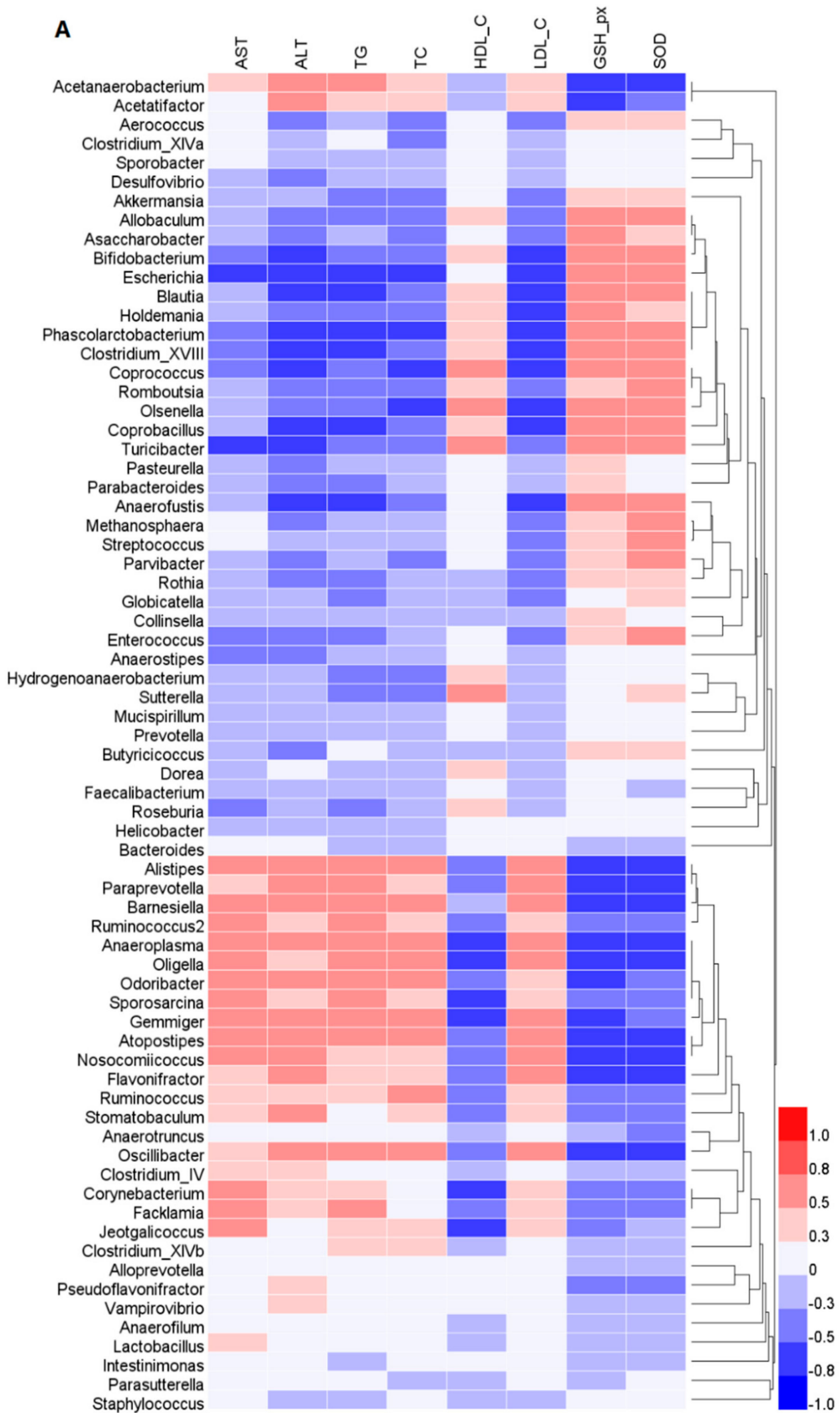

Figure 6. Cont. 


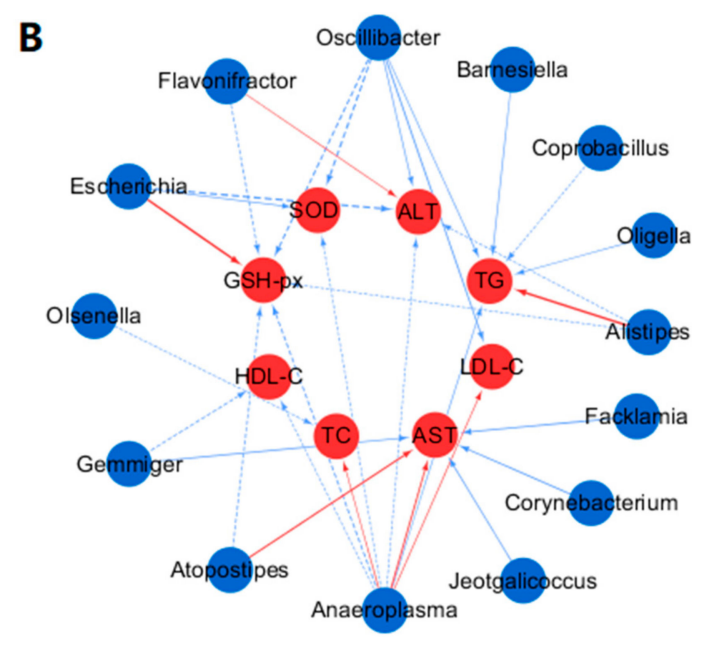

Figure 6. Spearman's correlations between the cecal microbiota and biochemical parameters measured in this study. (A) Heatmap of Spearman's correlation values showing the significant relationships between cecal microbiota and biochemical parameters. The color intensity represents the degree of association between cecal microbiota and biochemical parameters. (B) Visualization of the correlation network according to the significant correlations among the cecal microbiota and biochemical parameters. The significant edges were drawn in the network using the Spearman's correlation test $(|\mathrm{r}|>0.7)$. Each node represents a gut microbiota genus (blue nodes) and parameters (red nodes). The red and blue lines represent positive and negative correlations, respectively. In addition, the width of the lines indicates the strength of the correlation.

\section{Discussion}

Some studies have suggested that long-term HFD intake leads to TG, TC, and LDL-C synthesis rates exceeding the transport speed and metabolism in hepatocytes, inducing the occurrence of NAFLD [24]. HFD intake has been shown to be important in the development of NAFLD and is associated with increased oxidative stress and hepatic ectopic fat storage [25]. Notably, lipid accumulation in non-adipose tissues can lead to cellular dysfunction and the occurrence of NAFLD [26]. The abnormal oxidation of fatty acids results in reactive oxygen species formation, cell membrane fatty acid and phospholipid disruption, and cholesterol level changes, among other effects [25]. The most striking result of liver injury is the release of intracellular ALT and AST into the bloodstream, resulting in increased serum levels of these enzymes which reflect the extent of liver damage [27]. SOD and GSH-Px play vital roles in scavenging free radicals and maintaining the equilibrium of the redox state by synergistically acting on different parts of the free radical metabolic pathway to prevent oxidative damage [28-30].

Considering the side effects associated with many drugs, it is necessary to identify natural product-based drugs to combat NAFLD. Edible fungi, such as Grifola frondosa, have traditionally been used to treat human diseases. GFP is a natural heteropolysaccharide extracted from Grifola frondosa. In this study, animals administrated with GFP showed an obviously lower body weight when they were fed with HFD after both four and eight weeks $(p<0.01)$ (Table 1$)$. In addition, GFP reduced the concentrations of AST, TC, TG, and LDL-C and increased HDL-C, SOD, and GSH-Px concentrations compared with those of rats in the HFD group in serum or liver homogenates (Figure 1). The histological and pathological observations of the liver showed that GFP reduced hepatocyte steatosis compared with levels present in the HFD group $(p<0.01)$ (Figure 2). The above results indicate that the superior ability of GFP to attenuate NAFLD and obesity may be due to its profound and effective inhibition of fat accumulation induced by HFD.

We also investigated the expression levels of several genes related to oxidative stress, cytochrome P450 activation, bile acid anabolism, and inflammatory cytokine activity to explore the possible 
mechanism by which GFP may alleviate NAFLD [31,32]. A previous study showed that cytochrome P450 enzymes (CYPs) are associated with lipid peroxidation and damage to the integrity of cells. The transcriptional activation of CYP4A1 and CYP4A3 genes in AOX-/- mouse livers could lead to the metabolism of long-chain fatty acids into toxic dicarboxylic acids [33]. Accordingly, CYP4A2 was shown to be highly expressed in the liver tissues of rats with carbon tetrachloride-induced liver injury [34]. In our study, CYP4A1, CYP4A2, and CYP4A3 were found to be highly expressed in the livers of HFD rats, which may have caused liver cell and mitochondrial damage. The GFP group showed downregulation of the expression level of $C Y P 4 A 1$, but there was no difference regarding $C Y P 4 A 2$ and CYP4A3 expression compared with HFD group. CYP7A1 is the rate-limiting enzyme in the bile acid anabolism pathway, and the other bile acid anabolism pathway is an alternative pathway that is regulated by CYP8B1 [35]. Previously, the CYP7A1 mRNA levels of $\mathrm{db} / \mathrm{db}$ mice or animals fed HFD were shown to be downregulated [35,36]. In our experiment, CYP7A1 was downregulated in the HFD group compared with the NFD group. In contrast, CYP7A1 was significantly upregulated in the GFP group compared with the HFD group $(p<0.01)$. The increased CYP7A1 induced by GFP supplementation may regulate the conversion of cholesterol to bile acids [22]. Moreover, GFP administration effectively reduced the expression of ACC, TNF- $\alpha$, and SOCS2. The decrease in ACC gene expression could promote fat metabolism, thereby reducing TG levels in the liver [37]. TNF- $\alpha$ is considered to be one of the major inflammatory mediators found in NAFLD $[38,39]$. Compared with healthy subjects, patients with NASH showed overexpression of TNF- $\alpha$, but inhibition of TNF- $\alpha$ could significantly reduce aminotransferases levels [40]. Therefore, the suppression of TNF- $\alpha$ activation may be one of the mechanisms by which GFP protects hepatocyte membrane structure and function [21,41]. SOCS2 is an important regulator of hepatic lipid metabolism under HFD conditions. The concentration of TG is much lower in SOCS2 knockout mice than in wild-type mice that were fed HFD [42]. The reduction in SOCS2 gene expression following GFP administration shown in our study may be one of the mechanisms which promotes fat metabolism, thereby reducing liver lipid levels.

This study also aimed to determine the effect of GFP on modulating gut microbiota in HFD-fed rats by high-throughput next-generation $16 \mathrm{~S}$ rRNA gene sequencing. Gut microbes are vital to a host's health in terms of digesting complex carbohydrates, maintaining energy homeostasis, and regulating immune function, and they are correlated with the development of metabolic disorders, such as NAFLD and diabetes. Notably, the composition of gut microbiota varied after treatment with GFP. Compared with the HFD group, the GFP group showed a decrease in the Firmicutes-to-Bacteroidetes ratio. As reported in a previous investigation, Bacteroidetes, Firmicutes, and Actinobacteria are responsible for the degradation of complex, non-digestible polysaccharides [43]. An increased Firmicutes-to-Bacteroidetes ratio leads to a greater energy-harvesting capacity from undigested carbohydrates, producing more lipids [44]. Therefore, the decrease in liver steatosis observed in the GFP group may be related to the decrease in the Firmicutes-to-Bacteroidetes ratio. Moreover, the relative abandance of Allobaculum, Bacteroides, Bifidobacterium, Blautia, Coprococcus, Phascolarctobacterium, Prevotella, and Roseburia were significantly increased in the GFP group and the relative abandance of Acetatifactor, Alistipes, Flavonifractor, Paraprevotella, and Oscillibacter were significantly decreased in the GFP group compared with the HFD group. In addition, Acetatifactor, Flavonifractor, and Oscillibacter were shown to be significant components of the cecal microbiota according to the Spearman's correlation test between the gut microbiota and biomedical assays $(|r|>0.7)$. Therefore, Acetatifactor, Flavonifractor, and Oscillibacter may be very important in the improvement of NAFLD. Some studies have revealed that relative abundance of Allobaculum, Bacteroides, Bifidobacterium, Prevotella, and Roseburia is associated with a lean phenotype and that these genera are decreased in animals fed HFD [43-47]. A decrease in Phascolarctobacterium was correlated with NAFLD and T2D [48]. Moreover, a lower abundance of Bifidobacterium was correlated with liver injury and inflammation [46,49]. Some studies have reported that Roseburia, Coprococcus, and Blautia are also beneficial for host health [50,51]. In our study, supplementation with GFP significantly increased the proportions of Allobaculum, Bacteroides, Bifidobacterium, and other cecal microbiota, which may contribute to the beneficial effects of these 
treatments on the host by boosting the immune system and allowing it to fight against NAFLD. Recent studies have shown that Acetatifactor, Paraprevotella, and Oscillibacter are often increased in response to high-fat or high-sucrose diets [52-54]. An increase in Flavonifractor is associated with colorectal cancers [55] and displays invasive potential for background inflammatory bowel diseases. Alistipes was found to produce an unusual class of bacterial sulfonolipids, which increases in the mouse cecum in response to HFD. These results demonstrate that GFP, as a heteropolysaccharide, is a potential prebiotic that may modulate and maintain the microbial community, thus playing a vital role in relieving NAFLD. The results of this study may also serve as a novel experimental evidence of the ability to prevent and treat NAFLD through the diet. However, the modulation of expression of specific gene members related to lipid synthesis and conversion, cholesterol metabolism, and inflammation pathways were analyzed in this study, and the lack of global gene expression data may be causing some bias. The potential active mechanisms of GFP should be validated in further studies. In addition, the effects of gut microbiota on NAFLD are complex. Thus, the relationship between the gut microbiota and the positive effect of GFP in preventing NAFLD need to be investigated in further studies.

\section{Materials and Methods}

\subsection{Chemicals and Materials}

Fruiting bodies of Grifola frondosa were provided by the Qingyuan County of Zhejiang Province (Lishui, China). Chemicals and reagents were purchased from Sinopharm (Shanghai, China). Commercial test kits were obtained from the Jiancheng Bioengineering Company (Nanjing, China).

\subsection{Preparation of GFP}

Dried Grifola frondosa was chopped by an electric grinder and sieved through an 80 mesh to prepare dry Grifola frondosa powder. Grifola frondosa powder $(100 \mathrm{~g})$ was pretreated 5 times with $(v / w)$ $95 \%$ ethanol using ultrasonic extraction $\left(300 \mathrm{~W}, 50^{\circ} \mathrm{C}\right)$ for $60 \mathrm{~min}$. The precipitate was extracted 5 times with $(v / w) 55 \%$ ethanol using ultrasonic extraction $\left(300 \mathrm{~W}, 50^{\circ} \mathrm{C}\right)$ for $60 \mathrm{~min}$. Subsequently, the precipitate was extracted 10 times with hot water $(v / w)$ using ultrasonic extraction $\left(300 \mathrm{~W}, 80^{\circ} \mathrm{C}\right)$ for $60 \mathrm{~min}$. The supernatant was again precipitated with the addition of cold absolute ethanol to a final concentration of $80 \%(v / v)$ and kept at $4{ }^{\circ} \mathrm{C}$ overnight. The resulting precipitation was vacuum concentrated and freeze dried, yielding Grifola frondosa heteropolysaccharide (GFP).

\subsection{Experimental Animals and Study Design}

Male Wistar rats weighing $170 \pm 20 \mathrm{~g}$ were purchased from Shanghai SLAC Laboratory Animal Co., Ltd (Shanghai, China). The rats were kept under controlled conditions in a temperature range of $25 \pm 2{ }^{\circ} \mathrm{C}$ and a humidity level of $50 \pm 10 \%$ with a $12 \mathrm{~h}$ light/dark cycle. Rats were provided ad libitum access to commercial rodent chow and water.

After one week of adaptation to the environment, the rats were randomly divided into five groups of eight. Based on our previous preliminary experiments, in addition to water, the animals were given free access to a control diet (NFD, 13.5\% energy from fat; Lab Diet 1022; Lab Diet, Beijing, China), a high-fat diet (HFD, 67\% control diet, 20\% sucrose, 10\% lard, and 3\% cholesterol), or a high-fat diet with a daily gavage of GFP $(150 \mathrm{mg} / \mathrm{kg} \cdot \mathrm{bw}$ [9]. Body weight was measured at 0,4 , and 8 weeks during treatment. This article does not contain any studies with human participants performed by any of the authors. All procedures involving animals were conducted in strict accordance with the Chinese legislation on the use and care of laboratory animals and were approved by the Committee for Animal Experiments. The guidelines were established by the Institutional Animal Care and Use Committee of 900 Hospital of the Joint Logistics Team (Fuzhou General Hospital of Nanjing Military Command) (IACUC approval No. IACUC-2016-30, approved date: 5 July 2016). 


\subsection{Sample Collection}

The rats were anesthetized by an intraperitoneal injection of sodium pentobarbital ( $50 \mathrm{mg} \mathrm{kg}^{-1}$ body weight) at the end of the 8-week experimental period. Then, their abdominal cavity was opened and blood samples were collected by performing cardiac puncture. After $1 \mathrm{~h}$, the samples were centrifuged at $800 \mathrm{~g}$ for $10 \mathrm{~min}$ at $4{ }^{\circ} \mathrm{C}$, and the serum samples were subsequently stored. The livers were removed and rinsed with phosphate buffer saline. Partial tissues from the right lateral lobes of the livers were collected and fixed in $4 \%$ formaldehyde for histology analysis. Tissues from other partial livers were dissected, snap frozen in liquid nitrogen, and stored at $-80^{\circ} \mathrm{C}$ with the serum samples until use for further analysis.

\subsection{Biochemical Determination}

Serum was obtained by centrifugation of the blood samples at $3000 \mathrm{rpm}$ for $15 \mathrm{~min}$. Liver tissues were homogenized in a cold solution of $20 \mathrm{mM}$ Tris- $\mathrm{HCl}(\mathrm{pH} 7.4)(1: 10, w / v)$. The homogenate was centrifuged for $30 \mathrm{~min}$ at $2500 \mathrm{~g}$ [56]. The activities of serum ALT, serum AST, liver GSH-Px, and liverSOD and the levels of HDL-C, LDL-C, TG, and TC were detected using commercial test kits and instruments following the manufacturer's instructions.

\subsection{Histology Analysis}

The formalin-fixed livers from the rats were processed according to routine techniques, and $5 \mu \mathrm{m}$ thick paraffin sections were stained with hematoxylin and eosin for histological analysis [5].

\subsection{RNA Extraction and Real-Time Polymerase Chain Reaction}

Frozen liver tissues were homogenized and total RNA was extracted using TRIzol reagent (Invitrogen) in accordance with the manufacturer's instructions. Total RNA was extracted from the liver tissues using an RNA extraction kit (Code No. 9108/9109; Takara, Beijing, China). cDNA was synthesized using a PrimeScript ${ }^{\mathrm{TM}}$ RT Reagent Kit with gDNA Eraser (Code No. RR047A; Takara). RT-PCR was performed using SYBR Premix Ex Taq II (Code No. RR820A; Takara) in a $25 \mu \mathrm{L}$ reaction volume. Six selected candidate genes, CYP7A1, CYP8B1, CYP4A1, CYP4A2, CYP4A3, and suppressor of cytokine signaling 2 (SOCS2), tumor necrosis factor alpha (TNF- $\alpha$ ), and acetyl-CoA carboxylase (ACC) were assayed by qPCR [57]. The oligonucleotide primers used are shown in Table 3 . All reactions were run in triplicate. The conditions for the PCR were $95^{\circ} \mathrm{C}$ for $30 \mathrm{~s}$ followed by 45 cycles of $95^{\circ} \mathrm{C}$ for $5 \mathrm{~s}$, $57^{\circ} \mathrm{C}$ for $10 \mathrm{~s}$, and $72{ }^{\circ} \mathrm{C}$ for $15 \mathrm{~s}$ using an ABI PRISM 7300 thermal cycler.

Table 3. Sequences of primers used for RT-PCR in this study. ACC: acetyl-carboxylase; TNF- $\alpha$ : tumor necrosis factor alpha.

\begin{tabular}{ccc}
\hline Gene & Forward Primer & Reverse Primer \\
\hline$\beta$-actin & $5^{\prime}$-GGCACCACACTTTCTACAAT-3' & $5^{\prime}$-AGGTCTCAAACATGATCTGG-3' \\
CYP7A1 & $5^{\prime}$-GAGGGATTGAAGCACAAGAACC-3' & 5'-ATGCCCAGAGAATAGCGAGGT-3' $^{\prime}$ \\
CYP8B1 & $5^{\prime}$-CCCCTATCTCTCAGTACACATGG-3' & $5^{\prime}$-GACCATAAGGAGGACAAAGGTCT-3' \\
CYP4A1 & $5^{\prime}$-TTGAGCTACTGCCAGATCCCAC-3' & $5^{\prime}$-CCCATTTTTGGACTTCAGCACA-3' \\
CYP4A2 & $5^{\prime}$-CTCGCCATAGCCATGCTTATC-3' & $5^{\prime}$-CCTTCAGCTCATTCATGGCAATT-3' \\
CYP4A3 & $5^{\prime}$-CTCGCCATAGCCATGCTTATC-3' & $5^{\prime}$-CCTTCAGCTCATTCATGGCAATC-3' \\
SOCS2 & $5^{\prime}$-GGAACGGCACTGTTCACCTTTA-3' & $5^{\prime}$-AGCCTACAGAGATGCTGCAGAGA-3' \\
TNF- $\alpha$ & $5^{\prime}$-TGAACTTCGGGGTGATCGGT-3' & $5^{\prime}$-CTCCTCCGCTTGGTGGTTTG-3' \\
ACC & $5^{\prime}$-ACACTGGCTGGCTGGACAG-3' & $5^{\prime}$-CACACAACTCCCAACATGGTG-3' \\
\hline
\end{tabular}

\subsection{Cecal DNA Extraction and High-Throughput Sequencing}

Cecal DNA was prepared using a QIAamp-DNA Stool Mini Kit (Qiagen, Hilden, Germany) in accordance with the manufacturer's protocols. The DNA sequences of cecal contents were individually amplified with primer pairs targeting the 16S rRNA gene. The V3-V4 region of the 16S rRNA 
gene (V3-V4 hypervariable regions) was amplified by PCR using specific primers (forward primer: 5'-CCTACGGRRBGCASCAGKVRVGAAT-3'; reverse primer: 5'-GGACTACNVGGGTWTCTAATCC-3'). High-throughput sequencing was completed by Itechgene Technology Co., Ltd. (Shanghai, China).

\subsection{Statistical Analysis}

Statistical analysis was performed using one-way analysis of variance (ANOVA) and multiple comparisons were carried out to test for any significant differences among three groups with the SPSS software package, version 13.0. Values from each group were expressed as the mean \pm SD. $p<0.05$ and $p<0.01$ were considered to indicate statistical significance. The relative abundance of gut flora in different groups of rats was compared using R software (http://www.r-project.org/).

Author Contributions: The authors declare that there is no conflict of interest that could be perceived as prejudicing the impartiality of the research reported. X.L. performed the research work, curated the data, and wrote the original draft. F.Z. contributed to critically analyze the important data and reviewed the manuscript. B.L. and Y.H. designed the whole study, administrated the project, and reviewed the manuscript. All authors read and approved the final manuscript.

Funding: This work was funded by the National Natural Science Foundation of China (No. 31501432), the Fujian Science and Technology Plan project (No. 2017N5003), and Fujian Agriculture and Forestry University Science (No. CXZX2018055) and Technology Innovation Special Projects (No. CXZX2018056). Fujian Agriculture and Forestry University International Cooperation Project (No. KXG15001A), the National Science and Technology Support Program (2014BAD15B01).

Acknowledgments: The author Xin Li wish to thank Yongming Lin, Dan Liu and Hongpei Chen for instructing. The author also wish to thank to the Office of Scientific Research and Development, Fujian Agriculture and Forestry University for the administrative support.

Conflicts of Interest: The authors declare no conflicts of interest.

\section{References}

1. Sosa-Gutierrez, J.A.; Valdez-Solana, M.A.; Forbes-Hernandez, T.Y.; Avitia-Dominguez, C.I.; Garcia-Vargas, G.G.; Salas-Pacheco, J.M.; Flores-Herrera, O.; Tellez-Valencia, A.; Battino, M.; Sierra-Campos, E. Effects of Moringa oleifera Leaves Extract on High Glucose-Induced Metabolic Changes in HepG2 Cells. Biology 2018, 7, 37. [CrossRef] [PubMed]

2. Fu, J.H.; Sun, H.S.; Wang, Y.; Zheng, W.Q.; Shi, Z.Y.; Wang, Q.J. The Effects of a Fat- and Sugar-Enriched Diet and Chronic Stress on Nonalcoholic Fatty Liver Disease in Male Wistar Rats. Dig. Dis. Sci. 2010, 55, $2227-2236$. [CrossRef] [PubMed]

3. Gong, Z.; Tas, E.; Yakar, S.; Muzumdar, R. Hepatic lipid metabolism and non-alcoholic fatty liver disease in aging. Mol. Cell. Endocrinol. 2017, 455, 115-130. [CrossRef] [PubMed]

4. Forbes-Hernandez, T.Y.; Giampieri, F.; Gasparrini, M.; Afrin, S.; Mazzoni, L.; Cordero, M.D.; Mezzetti, B.; Quiles, J.L.; Battino, M. Lipid Accumulation in HepG2 Cells Is Attenuated by Strawberry Extract through AMPK Activation. Nutrients 2017, 9, E621. [CrossRef]

5. Byrne, C.D.; Targher, G. NAFLD: A multisystem disease. J. Hepatol. 2015, 62 (Suppl. 1), S47-S64. [CrossRef]

6. Vernekar, M.; Amarapurkar, D.; Joshi, K.; Singhal, R. Gene polymorphisms of desaturase enzymes of polyunsaturated fatty acid metabolism and adiponutrin and the increased risk of nonalcoholic fatty liver disease. Meta Gene 2017, 152-156. [CrossRef]

7. Boursier, J.; Mueller, O.; Barret, M.; Machado, M.; Fizanne, L.; Araujo-Perez, F.; Guy, C.D.; Seed, P.C.; Rawls, J.F.; David, L.A.; et al. The severity of NAFLD is associated with gut dysbiosis and shift in the metabolic function of the gut microbiota. Hepatology (Baltim. Md.) 2016, 63, 764-775. [CrossRef]

8. Chierico, D.; Nobili, V.; Vernocchi, P.; Russo, A.; de Stefanis, C.; Gnani, D.; Furlanello, C.; Zandonà, A.; Putignani, L.; Paci, P.; et al. Gut microbiota profiling of pediatric NAFLD and obese patients unveiled by an integrated meta-omics based approach. Hepatology 2017, 65, 451-464. [CrossRef]

9. Pan, Y.-Y.; Zeng, F.; Guo, W.-L.; Li, T.-T.; Jia, R.-B.; Huang, Z.-R.; Lv, X.-C.; Zhang, J.; Liu, B. Effect of Grifola frondosa $95 \%$ ethanol extract on lipid metabolism and gut microbiota composition in high-fat diet-fed rats. Food Funct. 2018, 9, 6269-6279. [CrossRef] 
10. Hu, R.; Guo, W.; Huang, Z.; Li, L.; Liu, B.; Lv, X. Extracts of Ganoderma lucidum attenuate lipid metabolism and modulate gut microbiota in high-fat diet fed rats. J. Funct. Foods 2018, 46, 403-412. [CrossRef]

11. Wang, X.; Shi, L.; Wang, X.; Feng, Y.; Wang, Y. MDG-1, an Ophiopogon polysaccharide, restrains process of non-alcoholic fatty liver disease via modulating the gut-liver axis. Int. J. Biol. Macromol. 2019. [CrossRef] [PubMed]

12. Zhu, H.; Wang, Z.; Wu, Y.; Jiang, H.; Zhou, F.; Xie, X.; Wang, R.; Hua, C. Untargeted metabonomics reveals intervention effects of chicory polysaccharide in a rat model of non-alcoholic fatty liver disease. Int. J. Biol. Macromol. 2019, 128, 363-375. [CrossRef] [PubMed]

13. Wu, Y.; Zhou, F.; Jiang, H.; Wang, Z.; Hua, C.; Zhang, Y. Chicory (Cichorium intybus L.) polysaccharides attenuate high-fat diet induced non-alcoholic fatty liver disease via AMPK activation. Int. J. Biol. Macromol. 2018, 118, 886-895. [CrossRef] [PubMed]

14. Wang, C.-M.; Yuan, R.-S.; Zhuang, W.-Y.; Sun, J.-H.; Wu, J.-Y.; Li, H.; Chen, J.-G. Schisandra polysaccharide inhibits hepatic lipid accumulation by downregulating expression of SREBPs in NAFLD mice. Lipids Health Dis. 2016, 15, 195. [CrossRef] [PubMed]

15. An, X.; Bao, Q.; Di, S.; Zhao, Y.; Zhao, S.; Zhang, H.; Lian, F.; Tong, X. The interaction between the gut Microbiota and herbal medicines. Biomed. Pharmacother. 2019, 118, 109252. [CrossRef]

16. Cotillard, A.; Kennedy, S.P.; Kong, L.C.; Prifti, E.; Pons, N.; le Chatelier, E.; Almeida, M.; Quinquis, B.; Levenez, F; Galleron, N.; et al. Dietary intervention impact on gut microbial gene richness. Nature 2013, 502, 585. [CrossRef]

17. Jayachandran, M.; Xiao, J.; Xu, B. A Critical Review on Health Promoting Benefits of Edible Mushrooms through Gut Microbiota. Int. J. Mol. Sci. 2017, 18, 1934. [CrossRef]

18. Chen, G.-T.; Ma, X.-M.; Liu, S.-T.; Liao, Y.-L.; Zhao, G.-Q. Isolation, purification and antioxidant activities of polysaccharides from Grifola frondosa. Carbohydr. Polym. 2012, 89, 61-66. [CrossRef]

19. Wang, C.L.; Meng, M.; Liu, S.B.; Wang, L.R.; Hou, L.H.; Cao, X.H. A chemically sulfated polysaccharide from Grifola frondosa induces HepG2 cell apoptosis by notch1-NF-kappa B pathway. Carbohydr. Polym. 2013, 95, 282-287. [CrossRef]

20. Chen, Y.; Liu, Y.; Sarker, M.M.R.; Yan, X.; Yang, C.; Zhao, L.; Lv, X.; Liu, B.; Zhao, C. Structural characterization and antidiabetic potential of a novel heteropolysaccharide from Grifola frondosa via IRS1/PI3K-JNK signaling pathways. Carbohydr. Polym. 2018, 198, 452-461. [CrossRef]

21. Yan, Z.; Fan, R.; Yin, S.; Zhao, X.; Liu, J.; Li, L.; Zhang, W.; Ge, L. Protective effects of Ginkgo biloba leaf polysaccharide on nonalcoholic fatty liver disease and its mechanisms. Int. J. Biol. Macromol. 2015, 80, 573-580. [CrossRef] [PubMed]

22. Lim, E.; Lim, J.Y.; Kim, E.; Kim, Y.-S.; Shin, J.-H.; Seok, P.R.; Jung, S.; Yoo, S.-H.; Kim, Y. Xylobiose, an Alternative Sweetener, Ameliorates Diabetes-Related Metabolic Changes by Regulating Hepatic Lipogenesis and miR-122a/33a in db/db Mice. Nutrients 2016, 8, 791. [CrossRef] [PubMed]

23. Kleiner, D.E.; Brunt, E.M.; van Natta, M.; Behling, C.; Contos, M.J.; Cummings, O.W.; Ferrell, L.D.; Liu, Y.C.; Torbenson, M.S.; Unalp-Arida, A.; et al. Design and validation of a histological scoring system for nonalcoholic fatty liver disease. Hepatology 2005, 41, 1313-1321. [CrossRef] [PubMed]

24. Matteoni, C.A.; Younossi, Z.M.; Gramlich, T.; Boparai, N.; Liu, Y.C.; McCullough, A.J. Nonalcoholic fatty liver disease: A spectrum of clinical and pathological severity. Gastroenterology 1999, 116, 1413-1419. [CrossRef]

25. Trauner, M.; Arrese, M.; Wagner, M. Fatty liver and lipotoxicity. Biochim. Et Biophys. Acta (Bba) Mol. Cell Biol. Lipids 2010, 1801, 299-310. [CrossRef]

26. Musso, G.; Gambino, R.; Cassader, M. Recent insights into hepatic lipid metabolism in non-alcoholic fatty liver disease (NAFLD). Prog. Lipid Res. 2009, 48, 1-26. [CrossRef]

27. Clark, J.M.; Brancati, F.L.; Diehl, A.M. The prevalence and etiology of elevated aminotransferase levels in the United States. Am. J. Gastroenterol. 2003, 98, 960-967. [CrossRef]

28. Cui, Y.; Cheng, Y.; Guo, Y.; Xie, Y.; Yao, W.; Zhang, W.; Qian, H. Evaluating the hepatoprotective efficacy of Aloe vera polysaccharides against subchronic exposure of aflatoxins B1. J. Taiwan Inst. Chem. Eng. 2017, 76, 10-17. [CrossRef]

29. Lv, L.; Cheng, Y.; Zheng, T.; Li, X.; Zhai, R. Purification, antioxidant activity and antiglycation of polysaccharides from Polygonum multiflorum Thunb. Carbohydr. Polym. 2014, 99, 765-773. [CrossRef]

30. Gu, X.; Manautou, J.E. Molecular mechanisms underlying chemical liver injury. Expert Rev. Mol. Med. 2012, 14, 1-25. [CrossRef] 
31. Tessari, P.; Coracina, A.; Cosma, A.; Tiengo, A. Hepatic lipid metabolism and non-alcoholic fatty liver disease. Nutr. Metab. Cardiovasc. Dis. 2009, 19, 291-302. [CrossRef] [PubMed]

32. Malaguarnera, M.; di Rosa, M.; Nicoletti, F.; Malaguarnera, L. Molecular mechanisms involved in NAFLD progression. J. Mol. Med. 2009, 87, 679-695. [CrossRef] [PubMed]

33. Hashimoto, T.; Fujita, T.; Usuda, N.; Cook, W.; Qi, C.; Peters, J.M.; Gonzalez, F.J.; Yeldandi, A.V.; Rao, M.S.; Reddy, J.K. Peroxisomal and mitochondrial fatty acid beta-oxidation in mice nullizygous for both peroxisome proliferator-activated receptor alpha and peroxisomal fatty acyl-CoA oxidase. Genotype correlation with fatty liver phenotype. J. Biol. Chem. 1999, 274, 19228-19236. [CrossRef] [PubMed]

34. Yang, S.-A.; Jung, Y.-S.; Lee, S.-J.; Park, S.-C.; Kim, M.-J.; Lee, E.-J.; Byun, H.-J.; Jhee, K.-H.; Lee, S.-P. Hepatoprotective effects of fermented field water-dropwort (Oenanthe javanica) extract and its major constituents. Food Chem. Toxicol. 2014, 67, 154-160. [CrossRef] [PubMed]

35. Li, T.; Owsley, E.; Matozel, M.; Hsu, P.; Novak, C.M.; Chiang, J.Y.L. Transgenic expression of cholesterol $7 \alpha$-hydroxylase in the liver prevents high-fat diet-induced obesity and insulin resistance in mice. Hepatology 2010, 52, 678-690. [CrossRef]

36. Wu, H.; He, K.; Wang, Y.; Xue, D.; Ning, N.; Zou, Z.; Ye, X.; Li, X.; Wang, D.; Pang, J. The antihypercholesterolemic effect of jatrorrhizine isolated from Rhizoma Coptidis. Phytomedicine 2014, 21, 1373-1381. [CrossRef]

37. Srivastava, R.A.K.; Pinkosky, S.L.; Filippov, S.; Hanselman, J.C.; Cramer, C.T.; Newton, R.S. AMP-activated protein kinase: An emerging drug target to regulate imbalances in lipid and carbohydrate metabolism to treat cardio-metabolic diseases. J. Lipid Res. 2012, 53, 2490-2514. [CrossRef]

38. Asrih, M.; Jornayvaz, F.R. Inflammation as a potential link between nonalcoholic fatty liver disease and insulin resistance. J. Endocrinol. 2013, 218, R25-R36. [CrossRef]

39. Tilg, H.; Diehl, A.M. Cytokines in alcoholic and nonalcoholic steatohepatitis. N. Engl. J. Med. 2000, 343, 1467-1476. [CrossRef]

40. Jung, S.H.; Park, H.S.; Kim, K.-S.; Choi, W.H.; Ahn, C.W.; Kim, B.T.; Kim, S.M.; Lee, S.Y.; Ahn, S.M.; Kim, Y.K.; et al. Effect of weight loss on some serum cytokines in human obesity: Increase in IL-10 after weight loss. J. Nutr. Biochem. 2008, 19, 371-375. [CrossRef]

41. Manco, M.; Marcellini, M.; Giannone, G.; Nobili, V. Correlation of serum TNF-alpha levels and histologic liver injury scores in pediatric nonalcoholic fatty liver disease. Am. J. Clin. Pathol. 2007, 127, 954-960. [CrossRef] [PubMed]

42. Hernandez, I.H.; Flores-Morales, A. SOCS2 deletion protects against hepatic steatosis but worsens insulin resistance in high-fat-diet-fed mice. Faseb J. 2012, 26, 3282-3291.

43. Scott, K.P.; Gratz, S.W.; Sheridan, P.O.; Flint, H.J.; Duncan, S.H. The influence of diet on the gut microbiota. Pharm. Res. 2013, 69, 52-60. [CrossRef] [PubMed]

44. Gangarapu, V.; Yildiz, K.; Ince, A.T.; Baysal, B. Role of gut microbiota: Obesity and NAFLD. Turk. J. Gastroenterol. 2014, 25, 133-140. [CrossRef]

45. Raza, G.S.; Putaala, H.; Hibberd, A.A.; Alhoniemi, E.; Tiihonen, K.; Makela, K.A.; Herzig, K.-H. Polydextrose changes the gut microbiome and attenuates fasting triglyceride and cholesterol levels in Western diet fed mice. Sci. Rep. 2017, 7, 1-11. [CrossRef]

46. Zhong, Y.; Marungruang, N.; Fåk, F.; Nyman, M. Effects of two whole-grain barley varieties on caecal SCFA, gut microbiota and plasma inflammatory markers in rats consuming low- and high-fat diets. Br. J. Nutr. 2015, 113, 1558-1570. [CrossRef]

47. Ghaffarzadegan, T.; Zhong, Y.; Fák Hállenius, F.; Nyman, M. Effects of barley variety, dietary fiber and $\beta$-glucan content on bile acid composition in cecum of rats fed low- and high-fat diets. J. Nutr. Biochem. 2018, 53, 104-110. [CrossRef]

48. Yan, H.; Lu, J.; Wang, Y.; Gu, W.; Yang, X.; Yu, J. Intake of total saponins and polysaccharides from Polygonatum kingianum affects the gut microbiota in diabetic rats. Phytomedicine 2017, 26, 45-54. [CrossRef]

49. Fang, D.Q.; Shi, D.; Lv, L.X.; Gu, S.L.; Wu, W.R.; Chen, Y.F.; Guo, J.; Li, A.; Hu, X.J.; Guo, F.F.; et al. Bifidobacterium pseudocatenulatum LI09 and Bifidobacterium catenulatum LI10 attenuate D-galactosamine-induced liver injury by modifying the gut microbiota. Sci. Rep. 2017, 7, 8770. [CrossRef]

50. Fu, X.; Cao, C.; Ren, B.; Zhang, B.; Huang, Q.; Li, C. Structural characterization and in vitro fermentation of a novel polysaccharide from Sargassum thunbergii and its impact on gut microbiota. Carbohydr. Polym. 2018, 183, 230-239. [CrossRef] 
51. Li, Y.; Xu, Q.; Huang, Z.; Lv, L.; Liu, X.; Yin, C.; Yan, H.; Yuan, J. Effect of Bacillus subtilis CGMCC 1.1086 on the growth performance and intestinal microbiota of broilers. J. Appl. Microbiol. 2016, 120, 195-204. [CrossRef] [PubMed]

52. Kong, C.; Gao, R.Y.; Yan, X.B.; Huang, L.S.; Qin, H.L. Probiotics improve gut microbiota dysbiosis in obese mice fed a high fat or high-sucrose diet. Nutrition 2019, 60, 175-184. [CrossRef] [PubMed]

53. Hibberd, A.A.; Yde, C.C.; Ziegler, M.L.; Honore, A.H.; Saarinen, M.T.; Lahtinen, S.; Stahl, B.; Jensen, H.M.; Stenman, L.K. Probiotic or synbiotic alters the gut microbiota and metabolism in a randomised controlled trial of weight management in overweight adults. Benef. Microbes 2019, 10, 121-135. [CrossRef] [PubMed]

54. Just, S.; Mondot, S.; Ecker, J.; Wegner, K.; Rath, E.; Gau, L.; Streidl, T.; Hery-Arnaud, G.; Schmidt, S.; Lesker, T.R.; et al. The gut microbiota drives the impact of bile acids and fat source in diet on mouse metabolism. Microbiome 2018, 6, 134. [CrossRef] [PubMed]

55. Ai, D.; Pan, H.; Han, R.; Li, X.; Liu, G.; Xia, L.C. Using Decision Tree Aggregation with Random Forest Model to Identify Gut Microbes Associated with Colorectal Cancer. Genes 2019, 10, 112. [CrossRef]

56. Dai, F.-J.; Hsu, W.-H.; Huang, J.-J.; Wu, S.-C. Effect of pigeon pea (Cajanus cajan L.) on high-fat diet-induced hypercholesterolemia in hamsters. Food Chem. Toxicol. 2013, 53, 384-391. [CrossRef]

57. Liang, J.; Deng, X.; Wu, F.S.; Tang, Y.F. Transcriptomic and proteomic analysis of human hepatic stellate cells treated with natural taurine. Mol. Med. Rep. 2013, 7, 1442-1452. [CrossRef]

(C) 2019 by the authors. Licensee MDPI, Basel, Switzerland. This article is an open access article distributed under the terms and conditions of the Creative Commons Attribution (CC BY) license (http://creativecommons.org/licenses/by/4.0/). 